\title{
Null-controllability, exact controllability, and stabilization of hyperbolic systems for the optimal time
}

\author{
Jean-Michel Coron ${ }^{1}$ and Hoai-Minh Nguyen ${ }^{2}$
}

\begin{abstract}
In this paper, we discuss our recent works on the null-controllability, the exact controllability, and the stabilization of linear hyperbolic systems in one dimensional space using boundary controls on one side for the optimal time. Under precise and generic assumptions on the boundary conditions on the other side, we first obtain the optimal time for the null and the exact controllability for these systems for a generic source term. We then prove the null-controllability and the exact controllability for any time greater than the optimal time and for any source term. Finally, for homogeneous systems, we design feedbacks which stabilize the systems and bring them to the zero state at the optimal time. Extensions for the non-linear homogeneous system are also discussed.
\end{abstract}

\section{INTRODUCTION}

Linear hyperbolic systems in one dimensional space are frequently used in modelling of many systems such as traffic flow, heat exchangers, and fluids in open channels. The stability and boundary stabilization of these hyperbolic systems have been studied intensively in the literature, see, e.g., [3] and the references therein. In this paper, we are interested in the null-controllability, the exact controllability, and the stabilization at finite time of linear hyperbolic systems in one dimensional space using boundary controls on one side. More precisely, we consider the system, for $(t, x) \in \mathbb{R}_{+} \times(0,1)$,

$$
\partial_{t} w(t, x)=\Sigma(x) \partial_{x} w(t, x)+C(x) w(t, x) .
$$

Here $w=\left(w_{1}, \cdots, w_{n}\right)^{\top}: \mathbb{R}_{+} \times(0,1) \rightarrow \mathbb{R}^{n}(n \geq 2), \Sigma$ and $C$ are $(n \times n)$ real matrix-valued functions defined in $[0,1]$. We assume that for every $x \in[0,1], \Sigma(x)$ is diagonal with $m \geq 1$ distinct positive eigenvalues, and $k=n-m \geq 1$ distinct negative eigenvalues. Using Riemann coordinates, one might assume that $\Sigma(x)$ is of the form

$$
\Sigma(x)=\operatorname{diag}\left(-\lambda_{1}(x), \cdots,-\lambda_{k}(x), \lambda_{k+1}(x), \cdots, \lambda_{n}(x)\right),
$$

where

$$
-\lambda_{1}(x)<\cdots<-\lambda_{k}(x)<0<\lambda_{k+1}(x)<\cdots<\lambda_{k+m}(x) .
$$

Throughout the paper, we assume that

$$
\lambda_{i} \text { is Lipschitz on }[0,1] \text { for } 1 \leq i \leq n(=k+m) .
$$

We also assume that

$$
C \in\left(L^{\infty}([0,1])\right)^{n \times n} .
$$

\footnotetext{
1 Jean-Michel Coron is with Sorbonne Universités, UPMC Univ Paris 06, UMR 7598, Laboratoire Jacques-Louis Lions, 4 place Jussieu, F-75252, Paris, France coroneann. jussieu.fr.

${ }^{2}$ Hoai-Minh Nguyen is with the Mathematics Department, Ecole Polytechnique Fédérale de Lausanne, EPFL, SB MATHAA CAMA, Station 8, CH-1015 Lausanne, Switzerland hoai-minh. nguyen@epfl. ch
}

We are interested in the following type of boundary conditions and boundary controls. The boundary conditions at $x=0$ are given by, for $t \geq 0$,

$$
\left(w_{1}, \cdots, w_{k}\right)^{\top}(t, 0)=B\left(w_{k+1}, \cdots, w_{k+m}\right)^{\top}(t, 0)
$$

for some $(k \times m)$ real constant matrix $B$, and the boundary controls at $x=1$ are, for $t \geq 0$,

$$
w_{k+1}(t, 1)=W_{k+1}(t), \quad \ldots, \quad w_{k+m}(t, 1)=W_{k+m}(t),
$$

where $W_{k+1}, \ldots, W_{k+m}$ are controls.

Let us recall that the control system (1), (6), and (7) is nullcontrollable (resp. exactly controllable) at the time $T>0$ if, for every initial data $w_{0}:(0,1) \rightarrow \mathbb{R}^{n}$ in $\left[L^{2}(0,1)\right]^{n}$ (resp. for every initial data $w_{0}:(0,1) \rightarrow \mathbb{R}^{n}$ in $\left[L^{2}(0,1)\right]^{n}$ and for every (final) state $w_{T}:(0,1) \rightarrow \mathbb{R}^{n}$ in $\left.\left[L^{2}(0,1)\right]^{n}\right)$, there is a control $W=\left(W_{k+1}, \ldots, W_{k+m}\right)^{\top}:(0, T) \rightarrow \mathbb{R}^{m}$ in $\left[L^{2}(0, T)\right]^{m}$ such that the solution of (1), (6), and (7) satisfying $w(t=$ $0, x)=w_{0}(x)$ vanishes (resp. reaches $\left.w_{T}\right)$ at the time $T: w(t=$ $T, x)=0$ (resp. $\left.w(t=T, x)=w_{T}(x)\right)$. Similar definitions hold for $w_{0} \in\left[L^{\infty}(0,1)\right]^{n}\left(\right.$ resp. $\left.w_{0}, w_{T} \in\left[L^{\infty}(0,1)\right]^{n}\right)$ with $W \in$ $\left[L^{\infty}(0, T)\right]^{m}$. Set

$$
\tau_{i}:=\int_{0}^{1} \frac{1}{\lambda_{i}(\xi)} d \xi \text { for } 1 \leq i \leq n .
$$

The exact controllability, the null-controllability, and the boundary stabilization problem of hyperbolic system in one dimension have been widely investigated in the literature for almost half a century. The pioneer works date back to Rauch and Taylor [28] and Russell [30]. In particular, it was shown, see [30, Theorem 3.2], that the system (1), (6), and (7) is null-controllable if for the time

$$
T \geq \tau_{k}+\tau_{k+1},
$$

and is exact controllable if $k=m$ and $B$ is invertible. The extension of this result for quasilinear system was initiated by Greenberg and Li [19] and Slemrod [31].

Concerning the stabilisation of (1), many articles are based on the boundary conditions with the following specific form

$$
\left(\begin{array}{l}
w_{-}(t, 0) \\
w_{+}(t, 1)
\end{array}\right)=G\left(\begin{array}{l}
w_{+}(t, 1) \\
w_{-}(t, 0)
\end{array}\right),
$$

where $G: \mathbb{R}^{n} \rightarrow \mathbb{R}^{n}$ is a suitable smooth vector field. Three approaches have been proposed to deal with (9). The first one is based on the characteristic method. This method was previously investigated in Greenberg and Li [19] for $2 \times 2$ systems and Qin [27] (see also Li [26]) for a generalization to $n \times n$ homogeneous nonlinear hyperbolic systems in the framework of $C^{1}$-norm. The second approach is based on 
Lyapunov functions see [7], [5] (see also [25], [6]). The third approach [11] is based on the study of delay equations works for $W^{2, p}$-norm with $p \geq 1$. These works typically impose restrictions on the magnitude of the coupling coefficients.

This restriction was overcome via backstepping approach. This was first proposed by Coron et al. [17] for $2 \times 2$ system $(m=k=1)$. Later this approach has been extended and now can be applied for general pairs $(m, k)$, see [18], [21], [1], [9]. In [17], the null-controllability is achieved via a feedback law for the time $\tau_{1}+\tau_{2}$ with $m=k=1$ via backstepping approach. In [21], the authors considered the case where $\Sigma$ is constant and obtained feedback laws for the null-controllability at the time

$$
T_{1}:=\tau_{k}+\sum_{l=1}^{m} \tau_{k+l} .
$$

It was later showed in [1], [9] that one can reach the nullcontrollability at the time

$$
T_{2}:=\tau_{k}+\tau_{k+1}
$$

Set

$$
T_{\text {opt }}:=\left\{\begin{array}{r}
\max \left\{\tau_{1}+\tau_{m+1}, \ldots, \tau_{k}+\tau_{m+k},\right. \\
\left.\tau_{k+1}\right\} \text { if } m \geq k, \\
\max \left\{\tau_{k+1-m}+\tau_{k+1}, \tau_{k+2-m}+\tau_{k+2},\right. \\
\left.\ldots, \tau_{k}+\tau_{k+m}\right\} \text { if } m<k .
\end{array}\right.
$$

In this paper, we report our recent works [13], [14], [15], [16] on the null-controllability, the exact controllability, and the stabilization of (1), (6), and (7). We show that the nullcontrollability holds at $T_{o p t}$ for generic $B$ and $C$ and the null-controllability holds for any $T>T_{o p t}$ under a precise condition on $B(B \in \mathscr{B}$ given in (13)), which holds for almost every matrix $k \times m$ matrix $B$. Similar conclusions holds for the exact controllability (with $B \in \mathscr{B}_{e}$ given in (14)) under natural, additional condition $m \geq k$. When the system is homogeneous, we show that the null-controllability is achieved via a time-independent feedback and there are Lyapunov's functions associated with these feedbacks. This result also holds for quasilinear setting. The starting point of our approach in the inhomogeneous case is the backstepping approach.

Remark 1.1: The backstepping approach for the control of partial differential equations was pioneered by Miroslav Krstic and his coauthors (see [24] for a concise introduction). The backstepping method is now frequently used for various control problems modeling by partial differential equations in one dimension. For example, it has been also used to stabilize the wave equation [23], [35], [32], the parabolic equations in [33], [34], nonlinear parabolic equations [36], and to obtain the null-controllability of the heat equation [12]. The standard backstepping approach relies on the Volterra transform of the second kind. It is worth noting that, in some situations, more general transformations have to be considered as for Korteweg-de Vries equations [4], KuramotoSivashinsky equations [10], Schrödinger's equation [8], and hyperbolic equations with internal controls [38].

\section{STATEMENT OF THE MAIN RESULTS}

Define

$\mathscr{B}:=\left\{B \in \mathbb{R}^{k \times m} ;(15)\right.$ holds for $\left.1 \leq i \leq \min \{k, m-1\}\right\}$,

and

$$
\mathscr{B}_{e}:=\left\{B \in \mathbb{R}^{k \times m} ; \text { (15) holds for } 1 \leq i \leq k\right\},
$$

where

the $i \times i$ matrix formed from the last $i$

columns and the last $i$ rows of $B$ is invertible.

We first show that the system (1), (6), and (7) is nullcontrollable for $B \in \mathscr{B}$ and is exact controllable for $B \in \mathscr{B}_{e}$ at the time $T_{\text {opt }}$ generically. More precisely, we have [13, Theorem 1.1]:

Theorem 2.1: Assume that (3) and (4) hold. We have

i) for each $B \in \mathscr{B}$, outside a discrete set of $\gamma$ in $\mathbb{R}$, the control system (1) with $C$ replaced by $\gamma C$, (6), and (7) is null-controllable at the time $T_{o p t}$.

ii) for each $\gamma$ outside a discrete set in $\mathbb{R}$, outside a set of zero measure of $B$ in $\mathscr{B}$, the control system (1) with $C$ replaced by $\gamma C,(6)$, and (7) is null-controllable at the time $T_{\text {opt }}$.

Remark 2.1: In the case $m=1$, we can show that there exists a (linear) time independent feedback which yields the null-controllability at the time $T_{o p t}$ for (1), (6), and (7) for all $B$, see [13, Theorem 1.1].

Remark 2.2: In the case $m=2$, we also established [13, Theorem 1.1] the following result on the optimality of $T_{o p t}$ : If $B \in \mathscr{B}, B_{k 1} \neq 0, \Sigma$ is constant, and $\left(T_{o p t}=\tau_{k}+\tau_{k+2}=\right.$ $\tau_{k-1}+\tau_{k+1}$ if $k \geq 2$ and $T_{o p t}=\tau_{1}+\tau_{3}=\tau_{2}$ if $k=1$ ), then there exists a non-zero constant matrix $C$ such that the system is not null-controllable at the time $T_{\text {opt }}$.

Concerning the exact controllability, we have

Theorem 2.2: Assume that $m \geq k \geq 1$, (3) and (4) hold. We have

i) for each $B \in \mathscr{B}_{e}$, outside a discrete set of $\gamma$ in $\mathbb{R}$, the control system (1) with $C$ replaced by $\gamma C$, (6), and (7) is exactly controllable at the time $T_{\text {opt }}$.

ii) for each $\gamma$ outside a discrete set in $\mathbb{R}$, outside a set of zero measure of $B$ in $\mathscr{B}_{e}$, the control system (1) with $C$ replaced by $\gamma C,(6)$, and (7) is exactly controllable at the time $T_{\text {opt }}$.

For the exact controllability and $T>T_{o p t}$, the generic assumption was removed in [22] (see also [14, Theorem 4]), where the following theorem is established

Theorem 2.3: Let $m \geq k \geq 1$. Assume that $B \in \mathscr{B}_{e}$ defined in (14). The control system (1), (6), and (7) is exactly controllable at any time $T$ greater than $T_{\text {opt }}$.

(In fact [22] gives the optimal time of exact controllability even if $B \notin \mathscr{B}_{e}$.)

For the null controllability and $T>T_{o p t}$, we removed the generic assumption was in [14, Theorems 2], where the following theorem is established 
Theorem 2.4: Let $k \geq m \geq 1$. Assume that $B \in \mathscr{B}$ defined in (13). The control system (1), (6), and (7) is nullcontrollable at any time $T$ greater than $T_{o p t}$.

Remark 2.3: Related controllability results can be also found in [20], [37].

Concerning the optimality of $T_{o p t}$, we can prove the following result [13, Proposition 1.6]

Proposition 2.1: Assume that $C \equiv 0$ and (15) holds for $1 \leq i \leq \min \{k, m\}$, then, for any $T<T_{o p t}$, there exists an initial datum such that $u(T, \cdot) \not \equiv 0$ for every control.

Remark 2.4: The controls used in Theorems 2.1 to 2.4 are of the form

$$
w_{+}(t, 1)=\sum_{r=1}^{R} A_{r}(t) w\left(t, x_{r}\right)+\int_{0}^{1} M(t, y) w(t, y) d y+h(t),
$$

where $R \in \mathbb{N}, A_{r}:[0, T] \rightarrow \mathbb{R}^{m \times n}, x_{r} \in[0,1](1 \leq r \leq R)$, $M:[0, T] \times[0,1] \rightarrow \mathbb{R}^{n \times n}$, and $h \in\left[L^{\infty}(0, T)\right]^{m}$. Moreover, the following conditions hold:

$$
\begin{gathered}
x_{r}<c<1 \text { for some constant } c \\
A_{r} \in\left[L^{\infty}(0, T)\right]^{n \times n}, \quad M \in\left[L^{\infty}((0, T) \times(0,1))\right]^{n \times n},
\end{gathered}
$$

for $1 \leq r \leq R$. The well-posedness of (1), (6), and (16) for broad solutions (see [13, Definition 3.1]) was established in [13, Lemma 3.2].

We next discuss homogeneous quasilinear hyperbolic systems. More precisely, we consider the equation, for $(t, x) \in$ $[0,+\infty) \times(0,1)$,

$$
\partial_{t} w(t, x)=\Sigma(x, w(t, x)) \partial_{x} w(t, x),
$$

instead of (1), and the boundary and control conditions (6) and (7). We assume that $\Sigma(x, y)$ for $x \in[0,1]$ and $y \in \mathbb{R}^{n}$ is of the form

$$
\begin{array}{r}
\Sigma(x, y)=\operatorname{diag}\left(-\lambda_{1}(x, y), \cdots,-\lambda_{k}(x, y),\right. \\
\left.\lambda_{k+1}(x, y), \cdots, \lambda_{k+m}(x, y)\right),
\end{array}
$$

where

$$
\begin{aligned}
-\lambda_{1}(x, y)<\cdots & <-\lambda_{k}(x, y) \\
& <0<\lambda_{k+1}(x, y)<\cdots<\lambda_{k+m}(x, y) .
\end{aligned}
$$

We assume, for $1 \leq i \leq n=k+m$,

$\lambda_{i}$ is of class $C^{2}$ with respect to $x$ and $y$

Concerning the quasilinear system (19), (6), and (7), we prove [15, Theorem 1.1]:

Theorem 2.5: Assume that $B \in \mathscr{B}$. For any $T>T_{\text {opt }}$, there exist $\varepsilon>0$ and a time-independent feedback control for (19), (6), and (7) such that if the compatibility conditions (at $x=0$ ) (23) and (24) below hold for $w(0, \cdot)$, and

$$
\|w(0, \cdot)\|_{C^{1}([0,1])}<\varepsilon
$$

then $w(T, \cdot)=0$.

The compatibility conditions considered in Theorem 2.1 are:

$$
w_{-}(0,0)=B\left(w_{+}(0,0)\right)
$$

and

$$
\begin{aligned}
& \Sigma_{-}(0, w(0,0)) \partial_{x} w_{-}(0,0) \\
& =B\left(w_{+}(0,0)\right) \Sigma_{+}(0, w(0,0)) \partial_{x} w_{+}(0,0)
\end{aligned}
$$

Here we denote

$$
\begin{gathered}
w_{-}=\left(w_{1}, \cdots, w_{k}\right)^{\top}, \quad w_{+}=\left(w_{k+1}, \cdots, w_{k+m}\right)^{\top} . \\
\Sigma_{-}(x, y)=\operatorname{diag}\left(-\lambda_{1}(x, y), \cdots,-\lambda_{k}(x, y)\right)
\end{gathered}
$$

and

$$
\Sigma_{+}(x, y)=\operatorname{diag}\left(\lambda_{k+1}(x, y), \cdots, \lambda_{n}(x, y)\right) .
$$

Remark 2.5: In [15], we also consider nonlinear boundary condition at $x=0$, i.e., instead of (6), we deal with

$$
w_{-}(t, 0)=\mathrm{B}\left(w_{+}(t, 0)\right) \text { for } t \geq 0
$$

for some

$$
\mathrm{B} \in\left(C^{2}\left(\mathbb{R}^{m}\right)\right)^{k} \text { with } \mathrm{B}(0)=0,
$$

Theorem 2.5 also holds if the condition $B \in \mathscr{B}$ is replaced by $\nabla B(0) \in \mathscr{B}$.

Remark 2.6: The feedbacks constructed in [15] use additional $4 m$ state-variables (dynamics extensions) to avoid imposing compatibility conditions at $x=1$.

In our recent work [16], we present Lyapunov's functions for the feedbacks given in Theorem 2.5 and use estimates for Lyapunov's functions to rediscover the finite stabilization result.

\section{THE IDEAS OF THE PROOF OF THEOREM 2.1-THEOREM 2.4}

The starting point of our analysis is the backstepping approach. The key idea of the backstepping approach is to make the following change of variables

$$
u(t, x)=w(t, x)-\int_{0}^{x} K(x, y) w(t, y) d y
$$

for some kernel $K: \mathscr{T} \rightarrow \mathbb{R}^{n \times n}$ which is chosen in such a way that the system for $u$ is easier to control. Here

$$
\mathscr{T}=\left\{(x, y) \in(0,1)^{2} ; 0<y<x\right\} .
$$

To determine/derive the equations for $K$, we first compute $\partial_{t} u(t, x)-\Sigma(x) \partial_{x} u(t, x)$. Taking into account (25), we formally have 1

$$
\begin{gathered}
\partial_{t} u(t, x)=\partial_{t} w(t, x)-K(x, x) \Sigma(x) w(t, x)+K(x, 0) \Sigma(0) w(t, 0) \\
+\int_{0}^{x}\left[\partial_{y}(K(x, y) \Sigma(y)) w(t, y)-K(x, y) C(y) w(t, y)\right] d y
\end{gathered}
$$

and

$\partial_{x} u(t, x)=\partial_{x} w(t, x)-\int_{0}^{x} \partial_{x} K(x, y) w(t, y) d y-K(x, x) w(t, x)$.

\footnotetext{
${ }^{1}$ We assume here that $u, w$, and $K$ are smooth enough so that the below computations make sense.
} 
It follows from (1) that

$$
\begin{gathered}
\partial_{t} u(t, x)-\Sigma(x) \partial_{x} u(t, x) \\
=(C(x)-K(x, x) \Sigma(x)+\Sigma(x) K(x, x)) w(t, x) \\
+K(x, 0) \Sigma(0) u(t, 0)+\int_{0}^{x}\left[\partial_{y} K(x, y) \Sigma(y)+K(x, y) \Sigma^{\prime}(y)\right. \\
\left.\quad-K(x, y) C(y)+\Sigma(x) \partial_{x} K(x, y)\right] w(t, y) d y .
\end{gathered}
$$

We search a kernel $K$ which satisfies the following two conditions

$$
\begin{aligned}
\partial_{y} K(x, y) \Sigma(y) & +\Sigma(x) \partial_{x} K(x, y) \\
& +K(x, y) \Sigma^{\prime}(y)-K(x, y) C(y)=0 \text { in } \mathscr{T}
\end{aligned}
$$

and, for $x \in(0,1)$,

$$
\mathscr{C}(x):=C(x)-K(x, x) \Sigma(x)+\Sigma(x) K(x, x)=0,
$$

so that one formally has

$$
\begin{aligned}
\partial_{t} u(t, x) & =\Sigma(x) \partial_{x} u(t, x) \\
& +K(x, 0) \Sigma(0) u(t, 0) \text { for }(t, x) \in \mathbb{R}_{+} \times(0,1) .
\end{aligned}
$$

Set

$$
Q:=\left(\begin{array}{cc}
0_{k} & B \\
0_{m, k} & I_{m}
\end{array}\right)
$$

and

$$
S(x):=K(x, 0) \Sigma(0) Q .
$$

Here and in what follows, $0_{i, j}$ denotes the zero matrix of size $i \times j$ for $i, j \in \mathbb{N}$, and $M_{p q}$ denotes the $(p, q)$-component of a matrix $M$. From (31), the matrix $S \in\left[L^{\infty}(0,1)\right]^{n \times n}$ has the structure

$$
S=\left(\begin{array}{cc}
0_{k, k} & S_{-+} \\
0_{m, k} & S_{++}
\end{array}\right)
$$

Using (6), equation (30) becomes, for $(t, x) \in \mathbb{R}_{+} \times(0,1)$,

$$
\partial_{t} u(t, x)=\Sigma(x) \partial_{x} u(t, x)+S(x) u(t, 0) .
$$

We are able to show that such a $K$ exists so that (34) holds [13, Lemma 3.3]; moreover, $K$ can be chosen in such a way that

$$
\left(S_{++}\right)_{p q}(x)=0 \text { for } 1 \leq q \leq p \leq m,
$$

this point turns out to be important for our analysis. It is shown in [13, Proposition 3.1] that the null-controllability and the exact controllability of (1), (6), and (7) at the time $T$ can be derived from the null-controllability and the exact controllability at the time $T$ of (34) equipped the boundary condition at $x=0$

$$
u_{-}(t, 0)=B u_{+}(t, 0) \text { for } t \geq 0,
$$

and the boundary controls at $x=1$

$$
u_{+}=U(t) \text { for } t \geq 0 \text { where } U \text { is the control. }
$$

In what follows, we discuss the idea of the proof of Theorem 2.1 and Theorem 2.4. The proofs of Theorem 2.2 and Theorem 2.3 (given in [14, Theorem 4]) are in the same spirit of these ones and not dealt with.

\section{A. On the proof of Theorem 2.1}

The idea of the proof is to derive the sufficient conditions for which the null-controllability holds. Using the characteristic method, these conditions will be written under the form $U+\mathscr{K} U=\mathscr{F}$ where $\mathscr{K}$ is an analytic compact operator with respect to $\lambda$ where $C$ is replaced by $\lambda C$. We then apply the Fredholm theory to obtain the conclusion. The process to derive the equation $U+\mathscr{K} U=\mathscr{F}$ is somehow involved. We refer the reader to [13] for the details.

\section{B. On the proof of Theorem 2.4}

As mentioned above the null-controllability of (1), (6), and (7) at the time $T$ is equivalent to the one (34)-(37) at the same time. The proof of the null-controllability of the later system is based on the Hilbert uniqueness method given in the following result [14, Lemma 1] whose proof is standard.

Lemma 3.1: Let $T>0$. System (34)-(37) is null controllable at the time $T$ if and only if, for some positive constant $C$,

$$
\int_{-T}^{0}\left|v_{+}(t, 1)\right|^{2} d t \geq C \int_{0}^{1}|v(-T, x)|^{2} d x \quad \forall v \in\left[L^{2}(0,1)\right]^{n},
$$

where $v(\cdot, \cdot)$ is the unique solution of the system, for $(t, x) \in$ $(-\infty, 0) \times(0,1)$,

$$
\partial_{t} v(t, x)=\Sigma(x) \partial_{x} v(t, x)+\Sigma^{\prime}(x) v(t, x),
$$

with, $t<0$,

$$
\begin{gathered}
v_{-}(t, 1)=0 \\
\Sigma_{+}(0) v_{+}(t, 0)=-B^{\top} \Sigma_{-}(0) v_{-}(t, 0) \\
+\int_{0}^{1} S_{-+}^{\top}(x) v_{-}(t, x)+S_{++}^{\top}(x) v_{+}(t, x) d x,
\end{gathered}
$$

and

$$
v(t=0, \cdot)=v \text { in }(0,1) .
$$

The next key part of the proof of Theorem 2.4 is the following compactness result for (39)-(41) [14, Lemma 4]; the structure of $S$ given in (35) plays a role in the proof.

Lemma 3.2: Let $k \geq m \geq 1, B \in \mathscr{B}$, and $T \geq T_{\text {opt }}$. Assume that $\left(v_{N}\right)$ be a sequence of solutions of (39)-(41) (with $v_{N}(0, \cdot)$ in $\left.\left[L^{2}(0,1)\right]^{n}\right)$ such that

$$
\begin{gathered}
\sup _{N}\left\|v_{N}(-T, \cdot)\right\|_{L^{2}(0,1)}<+\infty, \\
\lim _{N \rightarrow+\infty}\left\|v_{N,+}(\cdot, 1)\right\|_{L^{2}(-T, 0)}=0 .
\end{gathered}
$$

We have, up to a subsequence,

$$
v_{N}(-T, \cdot) \text { converges in } L^{2}(0,1),
$$

and the limit $V \in\left[L^{2}(0,1)\right]^{n}$ satisfies the equation

$$
V=\mathscr{K} V
$$

for some compact operator $\mathscr{K}$ from $\left[L^{2}(0,1)\right]^{n}$ into itself. Moreover, $\mathscr{K}$ depends only on $\Sigma, S$, and $B$; in particular, $\mathscr{K}$ is independent of $T$. 
Proof: [Proof of Theorem 2.4] For $T>T_{o p t}$, set

$$
Y_{T}:=\left\{V \in L^{2}(0,1): V \text { is the limit in } L^{2}(0,1)\right. \text { of }
$$

some subsequence of solutions $\left(v_{N}(-T, \cdot)\right)$

$$
\text { of (39)-(41) such that (43) and (44) hold }\} \text {. }
$$

It is clear that $Y_{T}$ is a vectorial space. Moreover, by (46) and the compact property of $\mathscr{K}$, we have

$$
\operatorname{dim} Y_{T} \leq C,
$$

for some positive constant $C$ independent of $T$.

We next show that

$$
Y_{T_{2}} \subset Y_{T_{1}} \text { for } T_{o p t}<T_{1}<T_{2} .
$$

Indeed, let $V \in Y_{T_{2}}$. There exists a sequence of solutions $\left(v_{N}\right)$ of (39)-(41) such that

$$
\left\{\begin{array}{c}
v_{N}(-T, \cdot) \rightarrow V \text { in } L^{2}(0,1), \\
\lim _{N \rightarrow+\infty}\left\|v_{N,+}(\cdot, 1)\right\|_{L^{2}\left(-T_{2}, 0\right)}=0 .
\end{array}\right.
$$

By considering the sequence $v_{N}(\cdot-\tau, \cdot)$ with $\tau=T_{2}-T_{1}$, we derive that $V \in Y_{T_{1}}$.

The arguments are then in the spirit of [2] (see also [29]) via an eigenvalue problem in finite dimension using a contradiction argument. By Lemma 3.1, to obtain the nullcontrollability at the time $T>T_{\text {opt }}$, it suffices to prove (38) by contradiction. Assume that there exists a sequence of solutions $\left(v_{N}\right)$ of (39)-(41) such that

$$
N \int_{-T}^{0}\left|v_{N,+}(t, 1)\right|^{2} d t \leq \int_{0}^{1}\left|v_{N}(-T, x)\right|^{2} d x=1 .
$$

By (45), up to a subsequence, $v_{N}(-T, \cdot)$ converges in $L^{2}(0,1)$ to a limit $V$. It is clear that $\|V\|_{L^{2}(0,1)}=1$; in particular, $V \neq 0$. Consequently,

$$
Y_{T} \neq\{0\} .
$$

By (48), (49), and (52), there exist $T_{o p t}<T_{1}<T_{2}<T$ such that

$$
\operatorname{dim} Y_{T_{1}}=\operatorname{dim} Y_{T_{2}} \neq 0 .
$$

We can prove that, for $V \in Y_{T_{1}}$,

$$
\Sigma \partial_{x} V+\Sigma^{\prime} V \text { is an element in } Y_{T_{1}} .
$$

Recall that $Y_{T_{1}}$ is real and of finite dimension. Consider its natural extension as a complex vectorial space and still denote its extension by $Y_{T_{1}}$. Define

$$
\begin{array}{cccc}
\mathscr{A}: & Y_{T_{1}} & \rightarrow & Y_{T_{1}} \\
V & \mapsto & \Sigma \partial_{x} V+\Sigma^{\prime} V .
\end{array}
$$

From the definition of $Y_{T_{1}}$, it is clear that, for $V \in Y_{T_{1}}$,

$$
V_{-}(1)=0
$$

and

$$
\begin{aligned}
\Sigma_{+}(0) V_{+}(0) & =-B^{\top} \Sigma_{-}(0) V_{-}(0) \\
& +\int_{0}^{1} S_{-+}^{\top}(x) V_{-}(x)+S_{++}^{\top}(x) V_{+}(x) d x .
\end{aligned}
$$

Since $Y_{T_{1}} \neq\{0\}$ and $Y_{T_{1}}$ is of finite dimension, there exists $\lambda \in \mathbb{C}$ and $V \in Y_{T_{1}} \backslash\{0\}$ such that

$$
\mathscr{A} V=\lambda V .
$$

Set

$$
v(t, x)=e^{\lambda t} V(x) \text { in }(-\infty, 0) \times(0,1) .
$$

Using (54) and (55), one can verify that $v(t, x)$ satisfies (39)(41). Applying the characteristic method, one can deduce that $v(t, \cdot)=0$ in $(0,1)$ for $t<-\tau_{k+1}-\cdots-\tau_{k+m}$. It follows that $V=0$ which contradicts the fact $V \neq 0$. Thus (38) holds and the null-controllability is valid for $T>T_{\text {opt }}$. The details can be found in [14].

\section{ON THE PROOF OF THEOREM 2.5}

We will also deal with the nonlinear boundary condition at $x=0$ as mentioned in Remark 2.5. We first consider the case $m>k$. Consider the last equation of (6) and impose the condition $w_{k}(t, 0)=0$. Using (15) with $i=1$ and the implicit function theorem, one can then write the last equation of (6) under the form

$$
w_{m+k}(t, 0)=M_{k}\left(w_{k+1}(t, 0), \cdots, w_{m+k-1}(t, 0)\right),
$$

for some $C^{2}$ nonlinear map $M_{k}$ from $U_{k}$ into $\mathbb{R}$ for some neighborhood $U_{k}$ of $0 \in \mathbb{R}^{m-1}$ with $M_{k}(0)=0$ provided that $\left|w_{+}(t, 0)\right|$ is sufficiently small.

Consider the last two equations of (6) and impose the condition $w_{k}(t, 0)=w_{k-1}(t, 0)=0$. Using (15) with $i=2$ and the Gaussian elimination approach, one can then write these two equations under the form (56) and

$$
w_{m+k-1}(t, 0)=M_{k-1}\left(w_{k+1}(t, 0), \cdots, w_{m+k-2}(t, 0)\right),
$$

for some $C^{2}$ nonlinear map $M_{k-1}$ from $U_{k-1}$ into $\mathbb{R}$ for some neighborhood $U_{k-1}$ of $0 \in \mathbb{R}^{m-2}$ with $M_{k-1}(0)=0$ provided that $\left|w_{+}(t, 0)\right|$ is sufficiently small, etc. Finally, consider the $k$ equations of (6) and impose the condition $w_{k}(t, 0)=\cdots=w_{1}(t, 0)=0$. Using (15) with $i=k$ and the Gaussian elimination approach, one can then write these $k$ equations under the form (56), (57), ..., and

$$
w_{m+1}(t, 0)=M_{1}\left(w_{k+1}(t, 0), \cdots, w_{m}(t, 0)\right),
$$

for some $C^{2}$ nonlinear map $M_{1}$ from $U_{1}$ into $\mathbb{R}$ for some neighborhood $U_{1}$ of $0 \in \mathbb{R}^{m-k}$ with $M_{1}(0)=0$ provided that $\left|w_{+}(t, 0)\right|$ is sufficiently small. These nonlinear maps $M_{1}, \ldots, M_{k}$ will be used in the construction of feedbacks.

Define

$$
\frac{d}{d t} x_{j}(t, s, \xi)=\lambda_{j}\left(x_{j}(t, s, \xi), w\left(t, x_{j}(t, s, \xi)\right)\right)
$$

with $x_{j}(s, s, \xi)=\xi$ for $1 \leq j \leq k$, and

$$
\frac{d}{d t} x_{j}(t, s, \xi)=-\lambda_{j}\left(x_{j}(t, s, \xi), w\left(t, x_{j}(t, s, \xi)\right)\right),
$$


with $x_{j}(s, s, \xi)=\xi$ for $k+1 \leq j \leq k+m$. We do not precise at this stage the domain of the definition of $x_{j}$. Later, we only consider the flows in the regions where the solution $w$ is well-defined.

To arrange the compatibility of our controls, we also introduce following auxiliary variables satisfying autonomous dynamics. Set $\delta=T-T_{\text {opt }}>0$. For $t \geq 0$, define, for $k+1 \leq j \leq k+m$,

$$
\zeta_{j}(0)=w_{0, j}(0), \zeta_{j}^{\prime}(0)=\lambda_{j}\left(0, w_{0}(0)\right) w_{0, j}^{\prime}(0),
$$

and

$$
\begin{gathered}
\eta_{j}(0)=1, \quad \eta_{j}^{\prime}(0)=0, \\
\zeta_{j}(t)=\eta_{j}(t)=0 \text { for } t \geq \delta / 2,
\end{gathered}
$$

The feedback is then chosen as follows:

$$
\begin{aligned}
& w_{m+k}(t, 1)=\zeta_{m+k}(t) \\
& +\left(1-\eta_{m+k}(t)\right) M_{k}\left(w_{k+1}\left(t, x_{k+1}\left(t, t+t_{m+k}, 0\right)\right), \ldots,\right. \\
& \left.w_{k+m-1}\left(t, x_{k+m-1}\left(t, t+t_{m+k}, 0\right)\right)\right)
\end{aligned}
$$

$$
\begin{aligned}
& w_{m+k-1}(t, 1)=\zeta_{m+k-1}(t) \\
+\left(1-\eta_{m+k-1}(t)\right) & M_{k-1}\left(w_{k+1}\left(t, x_{k+1}\left(t, t+t_{m+k-1}, 0\right)\right), \ldots\right. \\
& \left.w_{k+m-2}\left(t, x_{k+m-2}\left(t, t+t_{m+k-1}, 0\right)\right)\right)
\end{aligned}
$$

$\cdots$

$$
\begin{aligned}
& w_{m+1}(t, 1)=\zeta_{m+1}(t) \\
& +\left(1-\eta_{m+1}(t)\right) M_{1}\left(w_{k+1}\left(t, x_{k+1}\left(t, t+t_{m+1}, 0\right)\right), \ldots\right. \\
& \left.w_{m}\left(t, x_{m}\left(t, t+t_{m+1}, 0\right)\right)\right)
\end{aligned}
$$

and, for $k+1 \leq j \leq m$,

$$
w_{j}(t, 1)=\zeta_{j}(t)
$$

This feedback is well-determined by noting that (61) depends only on the current state, (62) depends only on the current state and (61), etc.

For $m \leq k$, the feedback law is given as follows:

$$
\begin{aligned}
& w_{m+k}(t, 1)=\zeta_{m+k}(t) \\
& +\left(1-\eta_{m+k}(t)\right) M_{k}\left(w_{k+1}\left(t, x_{k+1}\left(t, t+t_{m+k}, 0\right)\right), \ldots,\right. \\
& \left.w_{k+m-1}\left(t, x_{k+m-1}\left(t, t+t_{m+k}, 0\right)\right)\right) \text {, } \\
& \text {. } \\
& \begin{aligned}
w_{k+2}( & t, 1)=\zeta_{k+2}(t) \\
& +\left(1-\eta_{k+2}(t)\right) M_{2}\left(w_{k+1}\left(t, x_{k+1}\left(t, t+t_{k+2}, 0\right)\right)\right)
\end{aligned}
\end{aligned}
$$

and

$$
w_{k+1}(t, 1)=\zeta_{k+1}(t) .
$$

The null-controllability for small initial data can be derived from the properties of $M_{j}$ for $1 \leq j \leq k$. An key technical part of the proof is the well-posedness for (19) and (6) equipped these feedback laws, see [15, Lemma 2.2].

Conclusion: This paper is devoted to the null-controllability, exact controllability, and stabilization of hyperbolic systems for the optimal time. The starting point of the analysis in the inhomogeneous case is based on the backstepping approach. The ideas of the analysis are presented.

\section{REFERENCES}

[1] Jean Auriol and Florent Di Meglio, Minimum time control of heterodirectional linear coupled hyperbolic PDEs, Automatica J. IFAC 71 (2016), 300-307. MR 3521981

[2] Claude Bardos, Gilles Lebeau, and Jeffrey Rauch, Sharp sufficient conditions for the observation, control, and stabilization of waves from the boundary, SIAM J. Control Optim. 30 (1992), no. 5, 1024-1065. MR 1178650

[3] Georges Bastin and Jean-Michel Coron, Stability and boundary stabilization of 1-D hyperbolic systems, Progress in Nonlinear Differential Equations and their Applications, vol. 88, Birkhäuser/Springer, [Cham], 2016, Subseries in Control. MR 3561145

[4] Eduardo Cerpa and Jean-Michel Coron, Rapid stabilization for a Korteweg-de Vries equation from the left Dirichlet boundary condition, IEEE Trans. Automat. Control 58 (2013), no. 7, 1688-1695. MR 3072853

[5] Jean-Michel Coron, Georges Bastin, and Brigitte d'Andréa Novel, Dissipative boundary conditions for one-dimensional nonlinear hyperbolic systems, SIAM J. Control Optim. 47 (2008), no. 3, 1460-1498. MR 2407024

[6] Jean-Michel Coron, Brigitte d'Andréa Novel, and Georges Bastin, A lyapunov approach to control irrigation canals modeled by saintvenant equations, 1999 European Control Conference, 3173-3183.

[7] _ A strict Lyapunov function for boundary control of hyperbolic systems of conservation laws, IEEE Trans. Automat. Control 52 (2007), no. 1, 2-11. MR 2286756

[8] Jean-Michel Coron, Ludovick Gagnon, and Morgan Morancey, Rapid stabilization of a linearized bilinear 1-D Schrödinger equation, J. Math. Pures Appl. (9) 115 (2018), 24-73. MR 3808341

[9] Jean-Michel Coron, Long $\mathrm{Hu}$, and Guillaume Olive, Finite-time boundary stabilization of general linear hyperbolic balance laws via Fredholm backstepping transformation, Automatica J. IFAC 84 (2017), 95-100. MR 3689872

[10] Jean-Michel Coron and Qi Lü, Local rapid stabilization for a Korteweg-de Vries equation with a Neumann boundary control on the right, J. Math. Pures Appl. (9) 102 (2014), no. 6, 1080-1120. MR 3277436

[11] Jean-Michel Coron and Hoai-Minh Nguyen, Dissipative boundary conditions for nonlinear 1-D hyperbolic systems: sharp conditions through an approach via time-delay systems, SIAM J. Math. Anal. 47 (2015), no. 3, 2220-2240. MR 3356982

[12] Null controllability and finite time stabilization for the heat equations with variable coefficients in space in one dimension via backstepping approach, Arch. Rational Mech. Anal. 225 (2017), 9931023.

[13] _ Optimal time for the controllability of linear hyperbolic systems in one-dimensional space, SIAM J. Control Optim. 57 (2019), no. 2, 1127-1156. MR 3932617

[14] Null-controllability of linear hyperbolic systems in one dimensional space, https://arxiv.org/abs/1910.12268 (2019), submitted.

[15] _ Finite-time stabilization in optimal time of homogeneous quasilinear hyperbolic systems in one dimensional space, https://arxiv.org/abs/2005.13269 (2020), submitted.

[16] Lyapunov functions and finite time stabilization in optimal time for homogeneous linear and quasilinear hyperbolic systems, https://arxiv.org/abs/2007.04104 (2020), submitted.

[17] Jean-Michel Coron, Rafael Vazquez, Miroslav Krstic, and Georges Bastin, Local exponential $H^{2}$ stabilization of a $2 \times 2$ quasilinear hyperbolic system using backstepping, SIAM J. Control Optim. 51 (2013), no. 3, 2005-2035. MR 3049647

[18] Florent Di Meglio, Rafael Vazquez, and Miroslav Krstic, Stabilization of a system of $n+1$ coupled first-order hyperbolic linear PDEs with a single boundary input, IEEE Trans. Automat. Control 58 (2013), no. 12, 3097-3111. MR 3152271 
[19] J. M. Greenberg and Ta Tsien Li, The effect of boundary damping for the quasilinear wave equation, J. Differential Equations 52 (1984), no. 1, 66-75. MR 737964

[20] Long $\mathrm{Hu}$, Sharp time estimates for exact boundary controllability of quasilinear hyperbolic systems, SIAM J. Control Optim. 53 (2015), 3383-3410.

[21] Long Hu, Florent Di Meglio, Rafael Vazquez, and Miroslav Krstic, Control of homodirectional and general heterodirectional linear coupled hyperbolic PDEs, IEEE Trans. Automat. Control 61 (2016), no. 11, 3301-3314. MR 3571452

[22] Long $\mathrm{Hu}$ and Guillaume Olive, Minimal time for the exact controllability of one-dimensional first-order linear hyperbolic systems by one-sided boundary controls, Preprint, hal-01982662.

[23] Miroslav Krstic, Bao-Zhu Guo, Andras Balogh, and Andrey Smyshlyaev, Output-feedback stabilization of an unstable wave equation, Automatica J. IFAC 44 (2008), no. 1, 63-74. MR 2530469

[24] Miroslav Krstic and Andrey Smyshlyaev, Boundary control of PDEs, Advances in Design and Control, vol. 16, Society for Industrial and Applied Mathematics (SIAM), Philadelphia, PA, 2008, A course on backstepping designs. MR 2412038

[25] Guenter Leugering and E. J. P. Georg Schmidt, On the modelling and stabilization of flows in networks of open canals, SIAM J. Control Optim. 41 (2002), no. 1, 164-180. MR 1920161

[26] Ta Tsien Li, Global classical solutions for quasilinear hyperbolic systems, RAM: Research in Applied Mathematics, vol. 32, Masson, Paris; John Wiley \& Sons, Ltd., Chichester, 1994. MR 1291392

[27] Tie Hu Qin, Global smooth solutions of dissipative boundary value problems for first order quasilinear hyperbolic systems, Chinese Ann. Math. Ser. B 6 (1985), no. 3, 289-298, A Chinese summary appears in Chinese Ann. Math. Ser. A 6 (1985), no. 4, 514. MR 842971

[28] Jeffrey Rauch and Michael Taylor, Exponential decay of solutions to hyperbolic equations in bounded domains, Indiana Univ. Math. J. 24 (1974), 79-86. MR 361461

[29] Lionel Rosier, Exact boundary controllability for the Korteweg-de Vries equation on a bounded domain, ESAIM Control Optim. Calc. Var. 2 (1997), 33-55. MR 1440078

[30] David L. Russell, Controllability and stabilizability theory for linear partial differential equations: recent progress and open questions, SIAM Rev. 20 (1978), no. 4, 639-739. MR 508380

[31] M. Slemrod, Boundary feedback stabilization for a quasilinear wave equation, Control theory for distributed parameter systems and applications (Vorau, 1982), Lect. Notes Control Inf. Sci., vol. 54, Springer, Berlin, 1983, pp. 221-237. MR 793047

[32] Andrey Smyshlyaev, Eduardo Cerpa, and Miroslav Krstic, Boundary stabilization of a 1-D wave equation with in-domain antidamping, SIAM J. Control Optim. 48 (2010), no. 6, 4014-4031. MR 2645471

[33] Andrey Smyshlyaev and Miroslav Krstic, Closed-form boundary state feedbacks for a class of 1-D partial integro-differential equations, IEEE Trans. Automat. Control 49 (2004), no. 12, 2185-2202. MR 2106749

[34] On control design for PDEs with space-dependent diffusivity or time-dependent reactivity, Automatica J. IFAC 41 (2005), no. 9, 1601-1608. MR 2161123

[35] Boundary control of an anti-stable wave equation with antidamping on the uncontrolled boundary, Systems Control Lett. 58 (2009), no. 8, 617-623. MR 2542119

[36] Rafael Vazquez and Miroslav Krstic, Control of 1-D parabolic PDEs with Volterra nonlinearities. I. Design, Automatica J. IFAC 44 (2008), no. 11, 2778-2790. MR 2527199

[37] Norbert Weck, A remark on controllability for symmetric hyperbolic systems in one space dimension, SIAM J. Control Optim. 20 (1982), no. 1, 1-8. MR 642174

[38] Christophe Zhang, Finite-time internal stabilization of a linear 1-D transport equation, Preprint, hal-01980349 (2019). 